\title{
Simulating Vehicular Visible Light Communication: Physical Radio and MAC Modeling
}

\author{
Boris Tomaš \\ Faculty of Organization and Informatics, Computer Science and Information Engineering, \\ University of Zagreb, \\ Varaždin, Croatia \\ Email: boris.tomas@foi.hr
}

\author{
Mate Boban \\ NEC Laboratories Europe, \\ Heidelberg, Germany \\ Email:mate.boban@neclab.eu
}

\begin{abstract}
This paper presents work in progress on designing a transceiver model for realistic simulation of vehicular visible light communications (V2LC). We describe visible light communications (VLC) transceiver design and the most important operating procedures. We tested the VLC transceiver prototype design using a modified version of the 802.11 MAC protocol. Tests include three environments of different complexity levels that reflect relevant real life situations. Finally, we show preliminary results in the form of delay and packet delivery ratio.
\end{abstract}

\section{INTRODUCTION}

Visible light communications (VLC) is a novel communication technology that uses the terahertz-wide, unlicensed visible light spectrum. VLC has a great potential in supplementing or even replacing the existing radio frequency $(\mathrm{RF})$ communication technologies in application as diverse as device-to-device (D2D) communications, vehicle-to-vehicle (V2V) communications, and small-cell (e.g., femtocell) systems. Its physical characteristics are similar to infra-red (IR) communication; similar to IR, VLC also uses the spectrum that is under atmospheric window effect, thus making it highly sensitive to solar radiation noise.

In a vehicular communication setting, VLC is a particularly interesting communication technology, since most of the components that are needed to enable VLC are already part of modern vehicles. Specifically, an LED, LASER, or any other light emitting technology[1] that can be controlled electronically can be used as a VLC transmitter (Tx). Conveniently, headlights and taillights of modern vehicles contain LED lights, with LASER lights also being envisioned in the near future. Similarly, VLC receiver (Rx) component is usually either a photodiode (PD) [2], [3] or a CMOS camera [4], which can be found in many modern vehicles (e.g., a front camera for lane tracking or a rear camera for parking assistance).

Most of the existing network simulation environments assume that the underlying communication technology is operating in the RF range. Therefore, there is a lack of support in simulators for VLC, and even more so, for simulating VLC in vehicular environments. Since VLC and RF physical characteristics are significantly different, using VLC as the underlying communication technology instead of RF may render existing network protocols such as IEEE 802.11 (WiFi) protocol suite inoperable. Therefore, simulating IEEE 802.11 protocols over

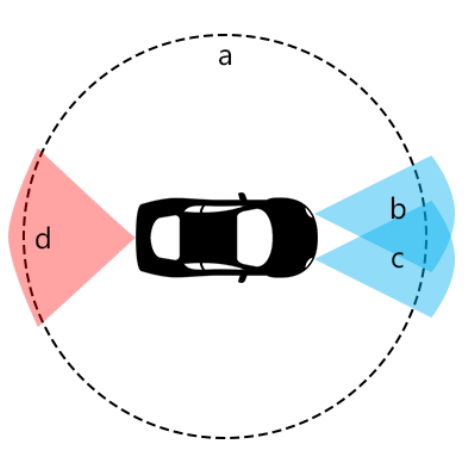

Fig. 1: Coverage of an omnidirectional radio frequency (RF) transceiver model (circle $a$ ) compared with the coverage of VLC transceiver (sectors $b, c$, and $d$ ).

VLC needs some modification of different characteristics of the VLC transceiver. Example in Fig. 1 shows the main difference between existing vehicular RF technology (used for, e.g., WiFi) with the coverage area $a$ and VLC. Specifically, VLC has a limited coverage area that can be separated into several sectors, which are not necessarily overlapping (e.g., sectors $b, c$, and $d$ ) Furthermore, while RF coverage described by circle $a$ applies for both RF transmitter (Tx) and receiver (Rx), for VLC transceiver Tx and Rx can and often do have different coverage, e.g., VLC Tx area is marked as $c$ and $b$, whereas Rx is marked as $d$. As shown in the figure, one vehicle can have multiple separated Tx and Rx, e.g., the figure shows two Tx and one Rx. Furthermore, VLC is highly sensitive to obstruction of the line-of-sight (LOS) between Tx and Rx, effectively making it a LOS-only communications technology, i.e., no behind-the-corner communications. On the other hand, RF can support communications in non-LOS scenarios. All of these facts indicate that i) the physical transceiver models designed for RF communication cannot be used for VLC; and ii) the protocols designed for RF communications need to be modified to work over VLC. 


\section{Network Simulation Environments: State of} THE ART

Network simulation environments are widely used by researchers and network system engineers to simulate computer networks, network topology and to predict network performance. Different types of wireless networks can be designed in various network simulators[5]. Basagni et al [6] performed a detailed analysis of existing network simulators; they concluded that there exists significant gap between simulation and experimental result, due to performance requirements. Also, most of the simulators lack proper or any implementation of:

- Carrier sensing

- Back-off timing

- Radio propagation model

- Interference detection

None of the widely used simulators (e.g., PhySim, NetSim, OPNET, GloMoSim, ns2, JiST/SWANS, PARSEC) support VLC communications. For this reason, we have designed a modular transceiver model for VLC simulation in JiST/SWANS ${ }^{1}$. JiST/SWANS ${ }^{2}$ is a high-performance discrete event simulation engine that runs in a standard Java virtual machine. It is a simulation system that executes discrete event simulations both efficiently and transparently by embedding simulation semantics [7]. JiST/SWANS lacks support for interference detection and has loose PHY model implementation that are trade-offs to increase performance [8]. It also lacks obstacle detection, i.e., whether Tx and Rx has LOS, and VLC support in general.

In following sections, we will introduce a new PHY layer transceiver model for VLC and evaluate the performance of the existing 802.11 MAC protocol (over VLC) with minor modifications.

\section{VLC PRopagation AND Physical LAYER Model}

Since a vehicular VLC system can have multiple Rx and Tx (e.g., front and rear cameras as Rx and multiple headlights and taillights as Tx), on the hardware level a VLC system will have to be able to internally address multiple Tx to send data and has to be able to receive data from multiple Rx. All incoming data streams should converge to a single transceiver device (which we call $V L C$ transceiver henceforth), thus having only one MAC address regardless of the number of Rx. Depending on the context, routing and network layers should either: i) not be aware of this configuration so they work (communicate) only with the single transceiver device to mimic "typical" RF communications; or ii) be able to send and receive data on different $\mathrm{Tx}$ and $\mathrm{Rx}$, thus exploiting this unique feature of VLC.

$V L C$ transceiver is aware of physical characteristics of each $\mathrm{Tx} / \mathrm{Rx}$ attached to it:

- Tx/Rx state (active or idle)

- Location of the node in the vehicle

\footnotetext{
${ }^{1}$ Simulator we developed is available here: https://github.com/boristomas/macvlc

${ }^{2}$ More info at jist.ece.cornell.edu
}

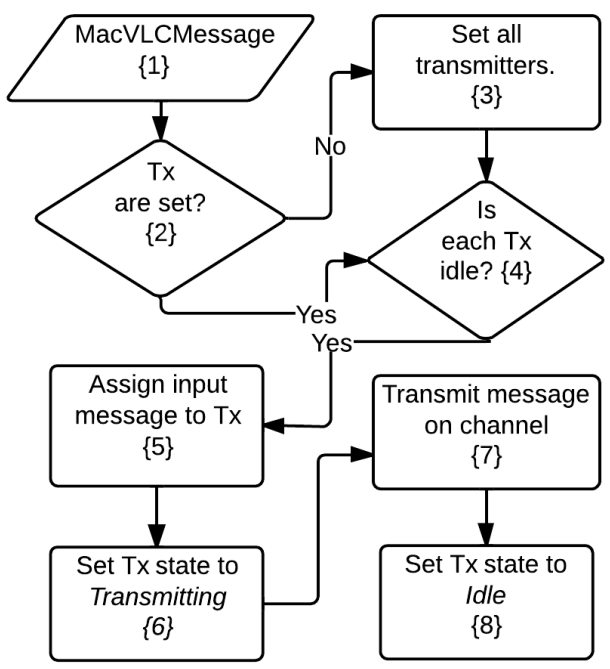

Fig. 2: VLC transceiver transmitting procedures

- Bearing (orientation of the optical transmitting or receiving component)

- Maximum sensing/transmitting range

- Angle of coverage (in Fig. 1, sectors $b$ and $c$ (Tx) and $d$ (Rx) can have different coverage angles)

A VLC transceiver provides these information to the MAC layer. It also uses notifications and events in the simulator so that the MAC protocol can process them. Some of the features that our VLC transceiver model implements include:

1) Multiple transmitters/receivers

2) Custom VLC path loss as defined in [9], [10]

3) Error notification is raised whenever a transceiver attempts to carry out unusual operation (e.g. attempting to send data via busy Tx)

4) Interference detection and notification is a feature used to provide more detailed information about the interference

\section{A. VLC transceiver transmitting procedure}

Fig. 2 shows the Tx procedures for a VLC transceiver: they start with incoming MAC VLC message (see Section IV-A). Each message can be assigned to any of the Tx in the $V L C$ transceiver. If no $\mathrm{Tx}$ is set then all available $\mathrm{Tx}$ are assigned by default (steps 2 and 3). For each assigned Tx, check if it is idle (step 4), if true then the message is assigned to Tx (step 5). If the assigned Tx is not idle (step 4), then it is skipped. On Idle Tx, it sets state to Transmitting (step 6). After the message is transmitted (step 7), it sets all participating Tx's states to Idle (step 8).

\section{B. VLC transceiver receiving procedure}

Fig. 3 shows the Rx procedures that start with packet received over the channel (step 1). JiST/SWANS simulator core implementation assumes that the communications is 


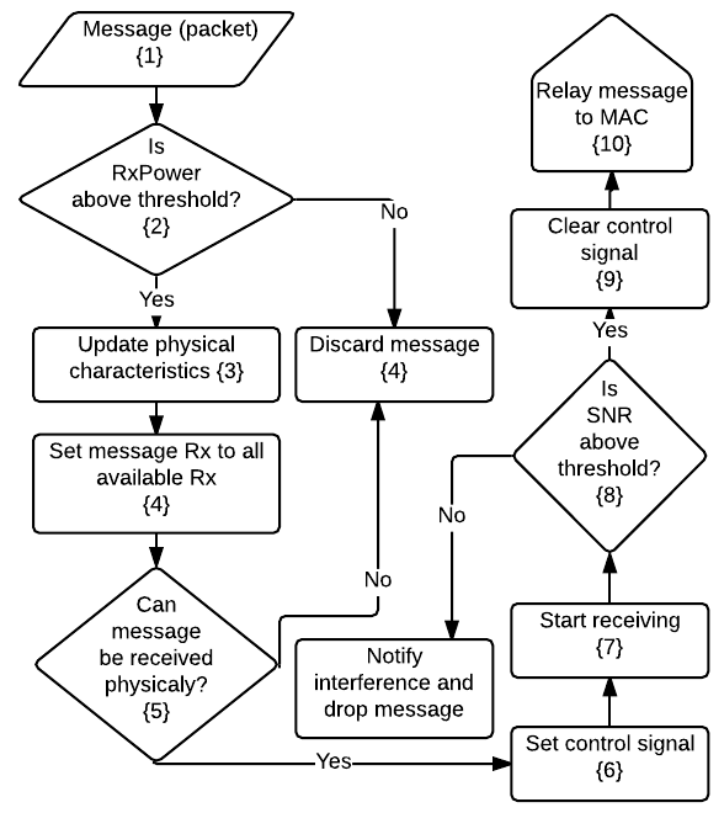

Fig. 3: VLC transceiver receiving procedure

omnidirectional. If the message's $\mathrm{Rx}$ power is below the transceiver sensitivity threshold then the message is discarded. Otherwise, message Rx-s are set to all available Rx (step 4) which are later filtered (step 5). After the message is received (step 7), SNR is calculated using additive model of all concurrent transmissions, as described in [11]. If SNR is above a certain threshold (see, e.g., [12]), the message can be received (i.e., only one message can be received in a single time period). If a message cannot be received due to low SNR, it is dropped; otherwise, it is relayed to the MAC layer.

Physical connectivity: Physical connectivity check (Fig. 3, step 5.) defines a set of physical conditions that need to be met so that two nodes can communicate. Because VLC communications is LOS-only, precise detection of LOS existence needs to be calculated in order to realistically simulate the VLC system. Simple scenario: two vehicles: A and B, each with only one Tx and and one Rx facing each other, can talk (in full duplex) only under the following physical conditions:

1) The distance between these two nodes is less than the max communication range;

2) Node A's Rx location is inside Nods B's Tx coverage;

3) Node B's Rx location is inside Node A's Tx coverage;

4) Signal strength between $A(T x)$ and $B(R x)$ or $A(R x)$ and $\mathrm{B}(\mathrm{Tx})$ is above specified threshold;

5) No other vehicle's outline shape (or any other object) intersects with line segments: $\overline{A(T x) B(R x)}$ and $\overline{B(T x) A(R x)}$.

If all above conditions are met, then the message from vehicle $\mathrm{B}$ is successfully received by vehicle A.
TABLE I: Simulation setup

\begin{tabular}{|c|c|}
\hline Simulation duration & $180 \mathrm{~s}$ \\
\hline Placement & see simulation scenarios \\
\hline Path loss exponent & 2.8 \\
\hline Vehicle length & $5 \pm 0.5$ \\
\hline Vehicle width & $1.7 \pm 0.3$ \\
\hline CBR $^{3}$ & 512 \\
\hline MAC protocol & 802.11 \\
\hline Tx \& Rx angle & $70^{\circ}$ \\
\hline Tx \& Rx LOS range & $30 \mathrm{~m}$ \\
\hline
\end{tabular}

\section{ModificATIONS TO IEEE 802.11 PROTOCOL STACK}

\section{A. MAC VLC message}

MAC message contains the following information:

- Transmission duration (Tx and $\mathrm{Rx}$ )

- Start transmission time (Tx and Rx)

- End transmission time (Tx and Rx)

- Interference state (Rx)

- Power level (Tx and Rx)

- Tx and Rx list

MAC protocol decides on which Tx to send the message; either all Tx-s or selected ones. When MAC VLC Message is received, its $\mathrm{Rx}$ list is populated with references to node $\mathrm{Rx}$ so that MAC can know which Rx actually received the message.

Because the current implementation of 802.11 MAC does not "know" how to work with VLC transceiver model and is not capable of full duplex communications, some modifications are necessary to enable IEEE 802.11 to support V2V VLC. When message is received from IEEE 802.11 MAC layer, all node's Tx are assigned to the message Tx list, because 802.11 has no Tx selection strategy. Furthermore, IEEE 802.11 does not support multiple concurrent Rx streams from multiple Rx-s; therefore, for VLC transceiver, we implemented concurrent correct reception from multiple physical Rx-s.

\section{Simulation}

We simulated three scenarios relevant to vehicular communications. The first scenario: Fig. 4.(a), there are only two nodes with a direct link and clear LOS. The second scenario (Fig. 4.(b)) has three nodes that can communicate with each other, where the vehicle in the middle uses both front and rear Tx and Rx. Final, third scenario: (Fig. 4.(c)) has three nodes where two nodes are not able to communicate (a hidden node scenario), and a third node that is able to communicate with the other two nodes. Simulation settings are shown in Table I.

\section{A. Metrics}

Metrics considered in this experiment are PDR (Package Delivery Rate) and average packet delay. Both metrics are calculated using time measured at:

- 802.11 MAC entry point at the junction with the upper layer on the transmitting node.

- 802.11 MAC exit point at the junction with upper layer on the receiving node.

\footnotetext{
${ }^{3}$ Constant Bit Rate
} 


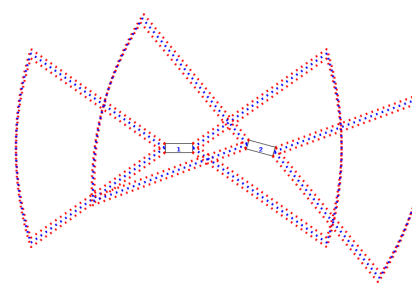

(a)

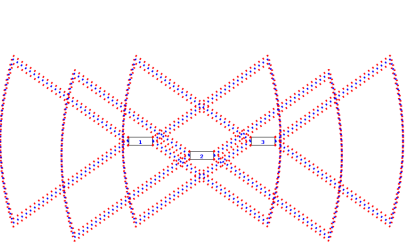

(b)

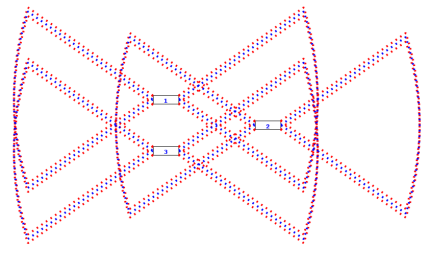

(c)

Fig. 4: Test scenarios. In all scenarios, the vehicle layout is static.

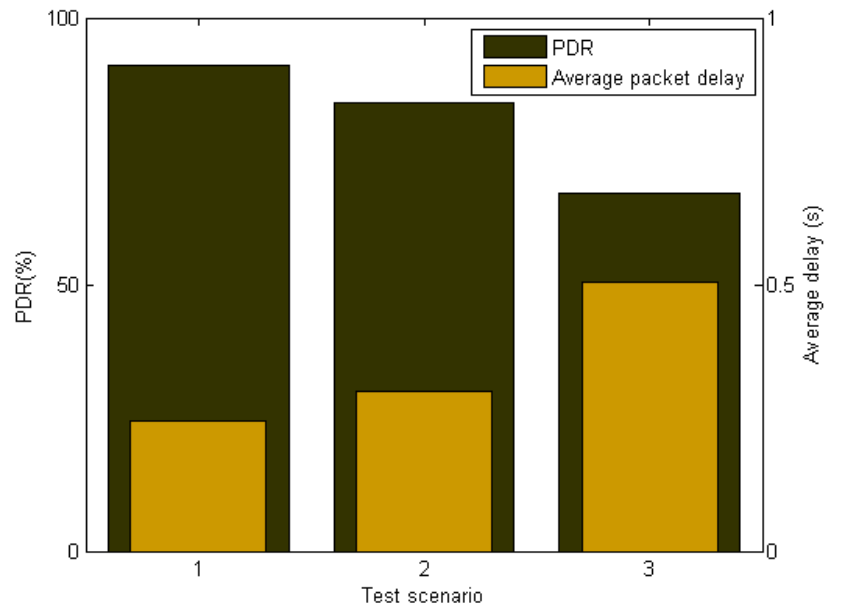

Fig. 5: 802.11 PDR and average packet delay

\section{Results AND CONCLUSION}

Each simulation scenario was run 6 times. For each run, results are shown in Fig. 5 as a dashed line. Averaged result is displayed as a solid line. Running unmodified, original IEEE 802.11 on VLC transceiver in any simulation scenario resulted with 0\% PDR and infinite message delay. This is the reason why modifications to the IEEE 802.11 were necessary in the first place. Fig. 5 shows results of modified IEEE 802.11 in defined simulation scenarios using PDR as a metric. It can be noticed that with rising simulation scenario complexity, PDR drops significantly. Also, it can be seen that, with the rising simulation scenario complexity, average delay increases. The results above show that performance of native IEEE 802.11 MAC protocol is in the acceptable range of 80-90\%[13] only for simple simulation scenarios. However, even with slightly more involved scenario (Fig. 4.(c)), the performance has significantly dropped, meaning that IEEE 802.11 MAC has decreasing performance with increasing network complexity. Although we already modified the original IEEE 802.11 to support VLC-specific communications, its performance would not be sufficient in complex, real-world scenarios. In other words, our results show that a novel MAC protocol specifically designed for VLC and take into account its specific physical properties is required. This MAC protocol need the following: enabling effective communications with multiple concurrent
Tx and Rx, taking into account the physical location of Tx and Rx sensors on the vehicle to make better medium access decisions, and make use of limited and geographicallypredetermined neighborhood location information.

To that end, our future research and experiments will include increasing $\mathrm{V} 2 \mathrm{~V}$ network complexity towards real case scenarios, including node mobility, and completely new MAC protocol designed specially for VLC V2V networks.

\section{ACKNOWLEDGMENT}

This work is supported in part by Ministry of Science and Technology, National Taiwan University and Intel Corporation under grants MOST-102-2911-I-002-001 and NTU103R7501.

\section{REFERENCES}

[1] E. Weidel, "Vehicle headlight," 2002.

[2] E. Pisek, S. Rajagopal, and S. Abu-Surra, "Gigabit rate mobile connectivity through visible light communication," IEEE International Conference on Communications (ICC), pp. 3122-3127, June 2012.

[3] S.-h. Yu, O. Shih, and H.-M. Tsai, "Smart automotive lighting for vehicle safety," IEEE Communications Magazine, no. December, pp. 50-59, 2013.

[4] T. Yamazato, I. Takai, H. Okada, T. Fujii, T. Yendo, S. Arai, M. Andoh, T. Harada, K. Yasautomi, K. Kagawa, and S. Kawahito, "Image-sensorbased visible light communication for automotive applications," IEEE Communications Magazine, no. July, pp. 88-97, 2014.

[5] H. Sundani, H. Li, V. Devabhaktuni, M. Alam, and P. Bhattacharya, "Wireless Sensor Network Simulators A Survey and Comparisons," International Journal Of Computer Networks (IJCN), vol. 2, pp. 249265, 2010.

[6] C. Vallati, V. Omwando, and P. Mohapatra, "Experimental work versus simulation in the study of mobile ad hoc networks," in Mobile Ad Hoc Networking: Cutting Edge Directions (S. Basagni, M. Conti, S. Giordano, and I. Stojmenovic, eds.), pp. 191-238, John Wiley \& Sons, Inc., 2 ed., 2013.

[7] R. Barr, Z. J. Haas, and R. V. Renesse, "Scalable wireless ad hoc network simulation," Ad hoc Wireless, and Peer-to-Peer Networks, 2005.

[8] E. B. Hamida, G. Chelius, and J. M. Gorce, "Impact of the Physical Layer Modeling on the Accuracy and Scalability of Wireless Network Simulation," Simulation, vol. 85, pp. 574-588, June 2009.

[9] B. Tomaš, "Visible Light Communication Physical Layer Design for JiST Simulation," Research papers, Faculty of Materials Science and Technology in Trnava, vol. 22, pp. 41-46, 2014.

[10] T. Komine and M. Nakagawa, "Fundamental analysis for visible-light communication system using LED lights," IEEE Transactions on Consumer Electronics, vol. 50, no. 1, pp. 100-107, 2004.

[11] W. Tranter and K. Kosbar, "Simulation of communication systems," IEEE Communications Magazine, vol. 32, pp. 26-35, July 1994.

[12] CISCO, Cisco Wireless Mesh Access Points, Design and Deployment Guide.

[13] M. Hassan, H. Vu, and T. Sakurai, "Performance analysis of the IEEE 802.11 MAC protocol for DSRC safety applications," IEEE Transactions on Vehicular Technology, vol. 60, no. 8, pp. 3882-3896, 2011. 